\title{
Cross-national differences in uncertainty avoidance predict the effectiveness of mass customization across East Asia: a large-scale field investigation
}

\author{
Emanuel de Bellis • Christian Hildebrand • \\ Kenichi Ito $\cdot$ Andreas Herrmann
}

Published online: 8 February 2015

(C) Springer Science+Business Media New York 2015

\begin{abstract}
Why does mass customization succeed in some East Asian markets but fail in others? Building on and extending prior work on uncertainty avoidance, this research suggests that providing mass customization in highly uncertainty-avoiding cultures can have negative consequences for consumers and companies, including longer configuration duration, lower conversion rates to actually purchase the customized product, and a reduced degree of sharing one's product with other consumers. We provide support for those propositions based on two large-scale field studies involving more than 700,000 prospective car buyers in Japan and Taiwan (scoring high on uncertainty avoidance) versus China and Singapore (scoring low on uncertainty avoidance). Our findings on actual customers suggest that neglecting cross-national differences in uncertainty avoidance across East Asia puts both consumers and companies at risk due to more onerous customization experiences for consumers and substantially lower conversion for companies.
\end{abstract}

Keywords Mass customization - Uncertainty avoidance - Cross-cultural marketing · Inter-Asian differences $\cdot$ Field study

\section{Introduction}

A new consumer trend in East Asia is a "quest for customized products and the thrill of 'co-creation' collaborations between consumers and product manufacturers" (Accenture 2012, p. 2). To be effective though, consumers need to have at least some

E. de Bellis $(\bowtie) \cdot$ C. Hildebrand $\cdot$ A. Herrmann

Center for Customer Insight, University of St. Gallen, Bahnhofstrasse 8, 9000 St. Gallen, Switzerland e-mail: emanuel.debellis@unisg.ch

K. Ito

Division of Psychology and Institute on Asian Consumer Insight, Nanyang Technological University, 50

Nanyang Avenue, Singapore 639798, Singapore 
insight into their own preferences when customizing products based on a large number of available features and combinations thereof (Simonson 2005). This research proposes that consumers' uncertainty involved in such a configuration process may have varying and sometimes unintended consequences among East Asian consumers given that East Asian cultures differ greatly in their degree of uncertainty avoidance (Hofstede et al. 2010). Differences in uncertainty avoidance have been shown to affect consumers' willingness to buy new product offerings, with high (vs. low) uncertaintyavoiding countries being more reluctant to adopt newly developed products and technologies (Lynn and Gelb 1996; van Everdingen and Waarts 2003; Yeniyurt and Townsend 2003). These findings are particularly important in a mass customization context where consumers face uncertainty with both the functionality of the product (given a typically more complex choice environment; Hildebrand et al. 2014) and their ability to contribute effectively during the configuration process.

Whether or not such differences in uncertainty avoidance affect people's consumption behavior beyond traditional comparisons between Eastern and Western cultures is widely unknown. The majority of cross-cultural research treated individuals from different East Asian countries as homogenous subjects of a largely identical group (e.g., Lehman et al. 2004) because researchers aimed to verify cultural frameworks developed in Western countries by testing them in East Asia. The resulting "East-West" comparisons, such as interdependent versus independent self-construal (Markus and Kitayama 1991) or holistic versus analytic cognition (Nisbett 2003), received tremendous research and media coverage during the past decades. In contrast, academic work on inter-Asian differences has attracted some attention in management and crosscultural psychology (e.g., Ang and Schmitt 1999; Cheng et al. 2014; Oyserman et al. 2002) but is still in its infancy in the area of marketing and consumer behavior (Schmitt 2013). Despite uncertainty avoidance being one of the most defining cultural differences between East Asian countries (Hofstede et al. 2010), research on the consequences of uncertainty avoidance on East Asians' consumption behavior is rare (see Frank et al. 2012 as a recent exception).

The current work addresses this lack of understanding within and across East Asian markets by presenting empirical evidence from two field studies examining the actual behavior of over 700,000 prospective car buyers. Our findings reveal that car buyers in Japan and Taiwan (scoring high on uncertainty avoidance) invest more time when customizing their preferred automobile relative to car buyers in China and Singapore (scoring low on uncertainty avoidance). Despite substantially longer configuration duration, consumers from high (vs. low) uncertainty-avoiding countries are less likely to actually buy the configured car. We also provide counter-intuitive evidence that Japanese (vs. Chinese) consumers share their car configurations less often with other consumers in spite of their distinct need to reduce uncertainty. These findings are put into perspective with regard to the high prevalence of taijin kyofusho in Japan (the concern to embarrass others) as well as the Japanese concept $w a$ (the tendency to maintain harmony among individuals).

Overall, our analyses of actual customers reveal highly distinct behaviors across four major East Asian countries. These findings have important implications for firms already operating in or considering entering markets in East Asia. In what follows, we develop four focal hypotheses that build on cross-national differences in uncertainty avoidance. We then present the results from two large-scale field studies and discuss the 
theoretical and managerial implications for both single-culture and cross-cultural marketing.

\section{Theoretical background and hypotheses}

While mass customization (MC) is becoming increasingly popular in East Asia (Accenture 2012), customizing a product for oneself involves a considerable degree of uncertainty during the configuration process. Dealing with a large number of product features, estimating the product's functionality based on the selected features and combinations thereof, and assessing others' approval of the customized product exposes a consumer to much more uncertainty than choosing a standard off-the-shelf product (Broniarczyk and Griffin 2014; Dellaert and Stremersch 2005; Hildebrand et al. 2014). A consumer's response to the uncertainty inherent in such a configuration process is critical in East Asia given that research from different domains revealed ample differences in uncertainty avoidance among East Asian countries (Hofstede et al. 2010; Quintal et al. 2010).

The cross-national concept of uncertainty avoidance is defined by the degree to which a country's residents deal with uncertainty regarding a future environment and is one of the five cultural dimensions that were proposed and extensively discussed by Hofstede and colleagues. In his classic field study on IBM employees in over 70 countries, Hofstede (2001) identified that individuals' stress levels at work strongly correlated with their perceived employment stability in the future and their unwillingness to violate company rules. Importantly, he showed that the strength of this correlation differs substantially across countries and argued that these differences stem from culture combatting individuals' tendencies to feel insecure about unknown future events. In delineating the concept of uncertainty avoidance, Hofstede (2001) distinguished uncertainty avoidance from risk avoidance. Whereas the perception of risk is related to a specific event to be feared, uncertainty is perceived when one is unable to foresee what will happen next. Other researchers conceptualized uncertainty avoidance as individuals' reliance on implicit or explicit rules, structures, and relationships to escape from the ambiguity in everyday life (Yaveroglu and Donthu 2002). For example, a country's dominance of religious beliefs is associated with the degree of uncertainty avoidance because it provides individuals in high uncertainty-avoiding countries with answers for many unanswerable questions that make people's lives ambiguous (e.g., life after inevitable death). Indeed, anthropologists showed that religiosity is more prominent in East Asian countries scoring high on uncertainty avoidance (e.g., Japan) relative to those scoring low (e.g., China; Hsu 1971).

Uncertainty avoidance has been suggested as one of the most defining cultural differences among East Asians (Frank et al. 2012; Hofstede et al. 2010). For instance, while Japanese, Taiwanese, Chinese, and Singaporeans are considered as both interdependent and holistic thinkers (Markus and Kitayama 1991; Nisbett 2003), they differ greatly from one another on the dimension of uncertainty avoidance. According to Hofstede et al.'s (2010) uncertainty avoidance index, Japanese (92 out of 100) and Taiwanese (69) score high on uncertainty avoidance while Chinese (30) and Singaporeans (8) score low on uncertainty avoidance. This central cultural difference has also been shown to affect individuals' consumption patterns. Consumers in high 
(vs. low) uncertainty-avoiding cultures tend to be less innovative and entrepreneurial, while their consumption is more conscientious (e.g., they consume less processed foods, buy more designer furniture, and choose mineral water over tap water; de Mooij and Hofstede 2002). Taken together, these characteristics of uncertainty avoidance suggest that individuals in high (vs. low) uncertainty-avoiding countries prefer a decision context that is free of potential ambiguity. In an MC context, in which clear decision strategies for one's preferred configuration are lacking, we predict that consumers in high (vs. low) uncertainty-avoiding countries take longer to configure their preferred product. More formally, we hypothesize:

H1: Prospective car buyers in high uncertainty-avoiding markets have a longer duration to complete the configuration process relative to prospective car buyers in low uncertainty-avoiding markets.

Hofstede (2001) and Hofstede et al. (2010) also proposed that uncertainty avoidance corresponds to consumers' level of anxiety toward an ambiguous, unpredictable, and uncertain future. For example, consumers in high (vs. low) uncertainty-avoiding countries value conservative over adventurous choices and are more ambiguityaverse to the extent that they hesitate to dive into a new and unknown situation. The inherent anxiety associated with uncertainty avoidance may have behavioral consequences for the effectiveness of MC offerings. For example, in the early 2000s, when the Internet was perceived as a new and technologically sophisticated medium associated with a considerable degree of uncertainty, consumers in high (vs. low) uncertaintyavoiding countries needed more reassurance and tools to reduce uncertainty in order to facilitate their online purchases (Singh et al. 2005). While customization has been present for centuries (e.g., home building or bespoke suits), consumers' ability to efficiently customize mass-produced consumer goods by making use of modern rendering technologies and choosing from a plethora of features is a dominant global trend across consumer markets (especially in East Asia; Accenture 2012). However, the uncertainty associated with such a feature-rich choice environment may have detrimental consequences for consumers high in uncertainty avoidance. Specifically, we propose:

H2: Prospective car buyers in high uncertainty-avoiding markets are less likely (a) to complete the configuration process and (b) to purchase the customized car relative to prospective car buyers in low uncertainty-avoiding markets.

How does configuration duration relate to one's probability to purchase a customized product? If, as proposed under H1, consumers from high (vs. low) uncertaintyavoiding countries invest more time into the configuration process, this may have consequences for their actual purchase probability as well. In the context of ecommerce websites, Sismeiro and Bucklin (2004) have shown that website visitors were less likely to buy a product when they returned to the retailer's website more frequently. While one could argue that longer configuration duration is related to larger product involvement, prior work has shown that longer task completion times are indicative of greater consumer uncertainty (Greenleaf and Lehmann 1995). Given these findings, we propose that configuration duration mediates the effect of cross-national 
differences in uncertainty avoidance on consumers' probability to purchase the customized product. Thus, we hypothesize:

H3: Longer configuration duration decreases the probability to purchase a customized car, resulting in lower conversion rates.

In today's competitive market environments, companies not only aim to increase the purchase probability of a focal consumer but also seek ways to maximize product diffusion and electronic word-of-mouth. In the light of the "interconnected, mobile [East Asian] consumer who wants to use consumer technology to make shopping fun, fast and collaborative" (Accenture 2012, p. 2), consumers' willingness to share their customized product with fellow consumers is particularly important in East Asia. A dominant finding in prior research is that uncertainty avoidance causes individuals to avoid social outings because of the fear of embarrassment and negative evaluation by others (Arrindell et al. 2004). In addition, uncertainty avoidance may strengthen the predisposition of culture-specific concepts of interpersonal relationships on consumers' advice-seeking behavior (Steenkamp et al. 1999). This is particularly important with respect to the Japanese culture because of the relatively high prevalence of taijin kyofusho (TKS), as individuals with TKS are highly concerned that their behavior will offend or embarrass another person (Hofmann et al. 2010). In such an environment, consumers may hesitate to seek advice from others and to share their configuration. Also, the meaning of acquiring and maintaining interpersonal relationships differs fundamentally across East Asia. For example, the Chinese concept guanxi is based on the exchange of utilitarian favors among individuals, while the Japanese concept wa refers to horizontal harmony among group members which is maintained through consensus building and avoidance of conflict (Cheng et al. 2014). From this perspective, asking others to comment on a customized product to achieve one's self-interests is likely to be socially acceptable in China but less so in Japan. Therefore, we propose:

H4: Prospective car buyers in high uncertainty-avoiding markets share their customized product less often with others relative to prospective car buyers in low uncertainty-avoiding markets.

The above hypotheses about the effects of cross-national differences in uncertainty avoidance on consumers' purchase and social sharing behavior are summarized in Fig. 1. Solid lines represent hypothesized effects while the dashed line indicates an exploratory effect. We do not provide a formal hypothesis (due to the lack of directionality) but will test the effect of social sharing on consumers' purchase probability in section 4.2 .

\section{Field study 1}

In collaboration with a large Western car manufacturer, we conducted a field study to examine whether consumers in high (vs. low) uncertainty-avoiding countries show longer configuration duration $(\mathrm{H} 1)$ and reduced configuration completion $(\mathrm{H} 2 \mathrm{a})$ when customizing their preferred car. 


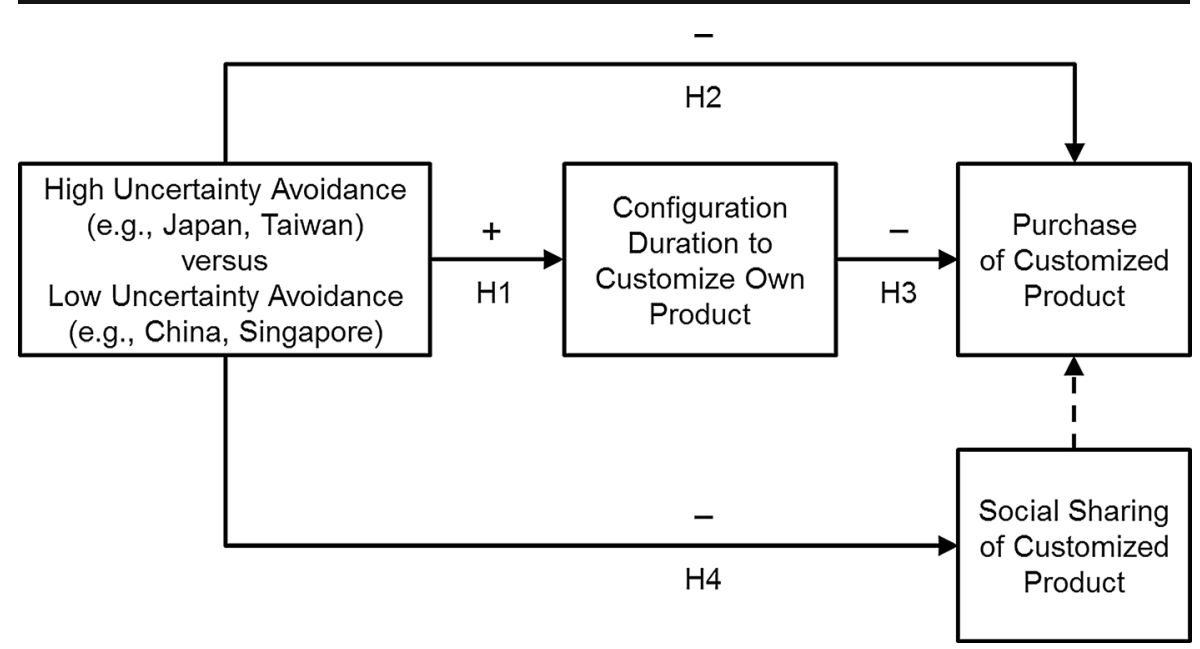

Fig. 1 Summary of hypotheses (solid lines are hypothesized; dashed line is exploratory)

\subsection{Data and measurement}

Across all analyzed markets, the car manufacturer employs exactly the same MC interface which guides consumers through the configuration process by letting them select each product attribute individually (i.e., one's preferred engine, exterior color, wheels, interior features, and add-ons), which has previously been conceptualized as by-attribute customization (Valenzuela et al. 2009). To test our hypotheses, the car manufacturer developed an MC tracking software that was incorporated in each market. When prospective car buyers entered the MC interface to configure their preferred car, the tracking software collected data on whether consumers completed their configuration (referred to as configuration completion) and the time span from starting the configuration until completion (referred to as configuration duration). Data were collected during 1 year (January 1 to December 31, 2013) for a total of 686,003 prospective car buyers who configured their preferred car in the Japanese, Taiwanese, Chinese, or Singaporean market.

\subsection{Results}

In line with our theorizing, we find that prospective car buyers in Japan and Taiwan (high in uncertainty avoidance) showed considerably longer configuration duration relative to those in China and Singapore (low in uncertainty avoidance). As such, Japanese and Taiwanese consumers took about $21 \mathrm{~min}$ to complete their car configuration whereas Chinese and Singaporean consumers only spent 19 and $14 \mathrm{~min}$, respectively $\left(M_{\text {Japan }}=20.94 \mathrm{~min}, M_{\text {Taiwan }}=21.16 \mathrm{~min}, M_{\text {China }}=18.88 \mathrm{~min}, M_{\text {Singapore }}=\right.$ $13.63 \mathrm{~min} ; F(1,442,697)=56.88, p<.001$; see Fig. 2a). In support of H1, post-hoc analyses reveal that all pairwise comparisons are significant $(p s<.001)$ except the one between the Japanese and Taiwanese markets $(p>.51)$. Interestingly, while investing more time, Japanese and Taiwanese consumers were less likely to complete their configuration. As shown in Fig. 2b, only 36.8 and $38.0 \%$ of prospective car buyers in Japan and Taiwan completed their car configuration relative to a significantly larger 
A



B

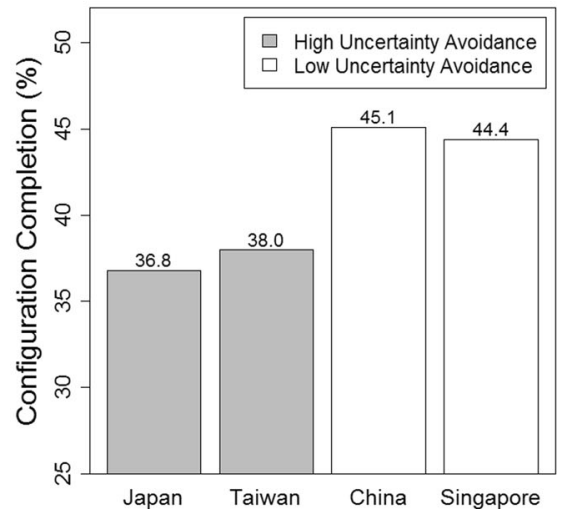

Fig. 2 Study 1: prospective car buyers in high uncertainty-avoiding markets (a) invested more time into the configuration process and (b) were less likely to complete it relative to those in low uncertainty-avoiding markets

fraction of 45.1 and $44.4 \%$ in China and Singapore, respectively $\left(\chi^{2}=4180, p<.001\right)$. Post-hoc analyses reveal that all pairwise comparisons are significant $(p \mathbf{S}<.001)$ apart from the one comparing the Chinese and the Singaporean markets $(p>.10)$. These results provide converging support for $\mathrm{H} 2 \mathrm{a}$.

In sum, we find that although prospective car buyers in Japan and Taiwan showed significantly longer configuration duration when customizing their preferred car, they were less likely to actually complete the configuration process relative to consumers in China and Singapore. Even though the reported effects are large and in line with our hypotheses, the aggregate nature of the data does not allow any inference on the individual level. To provide a remedy to this limitation, we conducted a follow-up study that enabled a more in-depth analysis of East Asians' distinct usage of MC interfaces.

\section{Field study 2}

To make inferences above and beyond the aggregate data of study 1 , we collaborated with the same car manufacturer as in study 1 and implemented an improved MC tracking software in the Japanese and Chinese markets, Asia's two largest economies and automobile markets. Study 2 examines the behavioral consequences of configuration duration (H3) and tests whether consumers in high (vs. low) uncertainty-avoiding countries show lower conversion rates $(\mathrm{H} 2 \mathrm{~b})$ and reduced social sharing behavior $(\mathrm{H} 4)$ when customizing their preferred car.

\subsection{Data and measurement}

This study allows an individual-level analysis of consumers' configuration processes by leveraging and combining dealer sales data (as indicated by an entry in a car dealer's database) with the behavioral data of the MC tracking software. Data sources were linked by unique identifiers of an individual car configuration via a user's device- 
specific hardware ID and a tracking code that is tied to a configuration and changes thereof. This adjustment enables the analysis of prospective car buyers' behavior over the time span from first visiting the MC interface until the final lead entry in the car dealer's database, which typically lasts several days or weeks (and is therefore referred to as overall configuration duration). Most importantly, we were able to analyze whether or not a configured car was purchased (referred to as conversion rate). In addition, the software tracked whether consumers sent their car configuration to a selfselected person via the manufacturer's MC interface (referred to as social sharing). Study 2 involves 78,978 car configurations from 42,074 prospective car buyers who had at least one but no more than 20 car configurations. ${ }^{1}$ Data were collected over a time span of 6 months (February 22 to September 1, 2014) in Japan and China.

\subsection{Results}

Similar to study 1, Japanese car buyers showed significantly longer overall configuration duration relative to Chinese car buyers. Specifically, analyzing those customers who returned at least once to the $\mathrm{MC}$ interface and eventually triggered a dealer request, Japanese (vs. Chinese) took five times as long from their first configuration until their final dealer configuration, with about 10 days in Japan relative to 2 days in China $\left(M_{\text {Japan }}=9.92\right.$ days, $M_{\text {China }}=1.73$ days; $\left.t(5282)=16.33, p<.001\right)$. Follow-up analyses revealed that Japanese customers had both higher return frequencies $\left(M_{\mathrm{Japan}}=3.49 \mathrm{vs}\right.$. $\left.M_{\text {China }}=3.16 ; t(5282)=4.31, p<.001\right)$ and longer time spans between two single configurations $\left(M_{\mathrm{Japan}}=68 \mathrm{~h}\right.$ vs. $\left.M_{\mathrm{China}}=13 \mathrm{~h} ; t(5282)=15.49, p<.001\right)$. These results provide additional support for $\mathrm{H} 1$.

In line with our previous findings on the behavioral consequences of uncertainty avoidance, consumers' configuration completion was significantly lower in Japan $(39.1 \%)$ compared to China $\left(59.8 \% ; \chi^{2}=12,827, p<.001\right)$, replicating the results from study 1 . Most importantly, conversion rates from a consumer's first configuration to his or her actual purchase differed greatly between markets with $18.3 \%$ (18,959 out of 103,578 started configurations) in China and only $2.3 \%$ (5803 out of 255,847 started configurations) in Japan $\left(\chi^{2}=29,554, p<.001\right)$. These results are summarized in Fig. 3 and provide strong support for $\mathrm{H} 2 \mathrm{a}$ and $\mathrm{H} 2 \mathrm{~b}$.

A logit model predicting consumers' conversion (non-purchase [coded as 0] vs. purchase [coded as 1]) based on their overall configuration duration reveals the expected negative effect: As prospective car buyers took longer for the configuration process, they were less likely to actually purchase the configured car $\left(\beta_{\text {configuration duration }}=-.21\right.$, $p<.001) .{ }^{2}$ Controlling for the respective market, both main effects are negative and significant $\left(\beta_{\text {configuration duration }}=-.09, p<.001 ; \beta_{\text {Japan vs. China }}=-1.18, p<.001\right)$, while their interaction is non-significant $\left(\beta_{\text {configuration duration } \times \text { Japan vs. China }}=.07, p>.10\right)$. In support of the proposed process explanation (see Fig. 1), a mediation model with

\footnotetext{
${ }^{1}$ A total of 318 consumers (or less than $1 \%$ of the sample) had more than 20 configurations and were likely to correspond to external dealer configurations, as noted by the company providing the data. Consequently, we removed these cases from all subsequent analyses. The reported results are robust and do not change if including these observations.

${ }^{2}$ Additional analyses indicate that both higher return frequencies $(\beta=-.46, p<.001)$ and longer time spans between two single configurations $(\beta=-.17, p<.001)$ are negatively related to purchase probability, in line with prior work (Greenleaf and Lehmann 1995; Sismeiro and Bucklin 2004).
} 


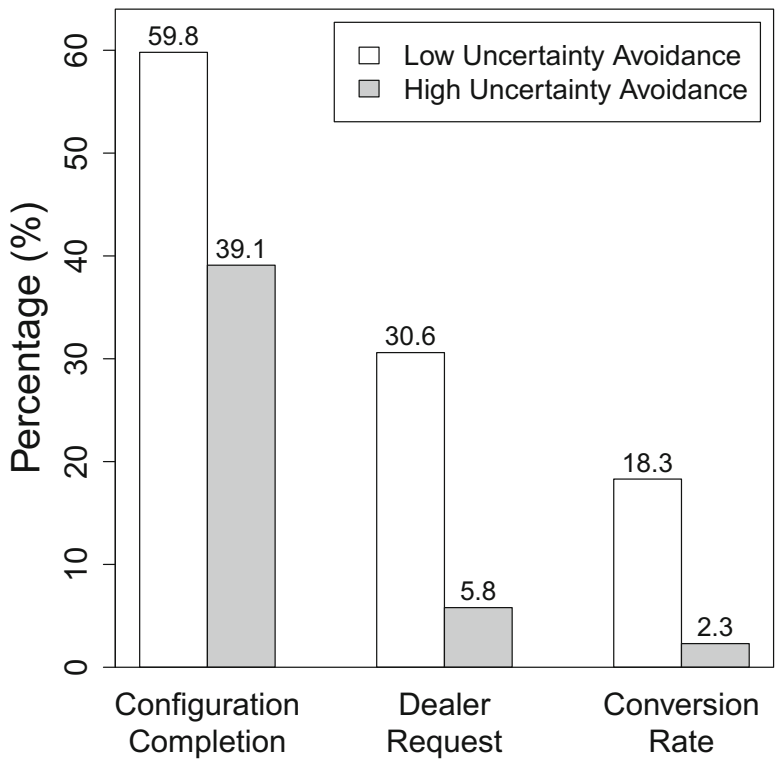

Fig. 3 Study 2: prospective car buyers in Japan (high in uncertainty avoidance) had a lower configuration completion rate, fewer dealer requests, and a reduced conversion rate relative to those in China (low in uncertainty avoidance)

bootstrapped estimates (Preacher and Hayes 2008) shows that the effect of high versus low uncertainty-avoiding markets on conversion rates is mediated by consumers' configuration duration $(\mathrm{a} \times \mathrm{b}=-.010$, with a $95 \%$ confidence interval excluding zero [ -.014 to -.005$])$, while the direct effect is reduced but yet significant $\left(\mathrm{c}^{\prime}=-1.20\right)$, indicating partial mediation. These results confirm H3 and demonstrate that Japanese consumers' increased time investment into the configuration process decreases (rather than increases) their tendency to actually purchase the customized car.

While investing more time into the configuration process, Japanese consumers were less likely to share their configuration with other consumers. In support of H4, only $0.13 \%$ of prospective car buyers in Japan shared their configuration relative to $0.28 \%$ in China $(t(42,072)=3.11, p<.001)$. Counter to intuition, sharing a configuration with others was associated with reduced conversion rates. Controlling for the respective market, both main effects are negative and significant $\left(\beta_{\text {social sharing }}=-.12, p<.001\right.$; $\left.\beta_{\text {Japan vs. China }}=-1.20, p<.001\right)$, while their interaction is non-significant $\left(\beta_{\text {social sharing } \times}\right.$ Japan vs. China $=-.08, p>.37)$. Given the increased attention of social sharing in recent consumer research, this finding highlights the need for further (both single-culture and cross-cultural) research on the consequences of social sharing and is discussed in more detail in section 5 .

\section{General discussion}

Based on a large-scale field investigation involving over 700,000 prospective car buyers in four major East Asian countries, the current research identified crossnational differences in uncertainty avoidance as an important driver of how consumers 
deal with MC offerings in East Asia. We showed that consumers in high uncertaintyavoiding markets (Japan and Taiwan) had substantially longer configuration duration, were less likely to purchase their customized car, and were less inclined to share their configuration with others relative to consumers in low uncertainty-avoiding markets (China and Singapore). These findings contribute to the emerging fields of research on $\mathrm{MC}$, socially enriched choice environments, and cross-cultural marketing, including the study of East Asian consumers.

While the majority of prior work on MC has examined outcome-related factors such as consumers' increased satisfaction with a customized product or their willingness to pay (e.g., Valenzuela et al. 2009), our findings suggest that consumers from high uncertainty-avoiding countries perceive customizing their own product as more onerous, with negative consequences such as ultimately lower conversion rates for companies. A similarly hot topic is the ubiquitous online social sharing activity with other consumers. This is the first series of studies demonstrating that social sharing is substantially reduced in high (vs. low) uncertainty-avoiding cultures. From an uncertainty avoidance standpoint, we suggest that this seemingly counter-intuitive finding can, at least partly, be explained by individuals' avoidance of social outings and their inherent wish not to offend others, which aligns with the prevalence of taijin kyofusho and the concept $w a$ in the Japanese culture. Importantly, while only few consumers shared their configuration with others, the negative effect of social sharing on consumers' purchase probability adds to the field of socially enriched choice environments and is in line with recent findings showing that the use of social media can have unintended, negative consequences for both consumers and firms (Hildebrand et al. 2013).

From a broader perspective on cross-cultural marketing, our work contributes to the development of research on the similarities and differences between East Asian societies. For more than 30 years, cross-cultural researchers have demonstrated the importance of culture on human cognition and behavior, primarily by comparing East Asians with North Americans or Western Europeans. This line of research has successfully established the perspective that one cannot comprehend consumption behavior independent of the cultural environment. However, the paradigm of East-West comparisons has been criticized for focusing mainly on Japan/China and the USA (Henrich et al. 2010) and for its failure to acknowledge differences within Eastern and Western cultures (Oyserman et al. 2002). The current findings contribute to more nuanced cross-cultural research, as they highlight the behavioral consequences of (neglecting) cultural differences within East Asia. In particular, we built on the concept of uncertainty avoidance - the degree to which a society deals with uncertainty regarding the future (Hofstede 2001) — as a defining characteristic to aid in understanding East Asian cultures. In line with recent work of Frank and colleagues (e.g., Frank et al. 2012), we demonstrated that East Asian markets and their consumers' behavior differ greatly, with important consequences for firms operating in or considering entering these markets.

Despite these contributions, we acknowledge the limitations inherent in using industry field data. As such, we have not experimentally manipulated uncertainty avoidance to isolate the proposed effects in a more controlled setting. However, given prior work on uncertainty avoidance in East Asia (Frank et al. 2012; Hofstede et al. 2010) and the large datasets used in this research, we believe that the current results are robust. In addition, the analysis of four different countries controls for potentially 
confounding factors arising from differences on economic or other cultural dimensions. For instance, GDP per capita is comparable between Japan, Taiwan, and Singapore, while China and Taiwan share a similar historical background. As such, the results of this large-scale field investigation hold even after controlling for other major differences at the country level.

With respect to future directions, one particularly puzzling observation relates to Japanese consumers being perceived as technology mavens with a strong affection for new technologies and unique products. The present research provides a more nuanced observation and suggests that using advanced $\mathrm{MC}$ interfaces does not equal actual purchase. Future research may examine the antecedents that cause such discrepancies as well as potential strategies to assist consumers from high uncertainty-avoiding cultures when navigating through potentially complex choice environments, such as by-alternative instead of by-attribute customization (Valenzuela et al. 2009) or customization via starting solutions (Hildebrand et al. 2014). Besides contributing to the emerging field of consumer decision-making in electronically enhanced choice environments, such research would enhance our understanding of the behavioral consequences of East Asia's crave for customized products.

Acknowledgments The authors gratefully acknowledge the guidance of the editor and the two reviewers. They also wish to thank the car manufacturer for providing the data, Lucas Beck for assisting with the data coding, as well as Anna-Louise McCaskey for proofreading the manuscript.

\section{References}

Accenture (2012). Asia consumer product trends: implications for retailers and manufacturers. www. accenture.com/us-en/Pages/insight-asia-consumer-product-trends-implications-retailers-manufacturers. aspx. Accessed 1 July 2014.

Ang, S. H., \& Schmitt, B. H. (1999). Introduction to special issue. Asia Pacific Journal of Management, 16, 3-8.

Arrindell, W. A., Eisemann, M., Oei, T. P. S., Caballo, V. E., Sanavio, E., Sica, C., et al. (2004). Phobic anxiety in 11 nations: part II. Hofstede's dimensions of national cultures predict national-level variations. Personality and Individual Differences, 37, 627-643.

Broniarczyk, S. M., \& Griffin, J. (2014). Decision difficulty in the age of consumer empowerment. Journal of Consumer Psychology 24, 608-625.

Cheng, B., Boer, D., Chou, L., Huang, M., Yoneyama, S., Shim, D., et al. (2014). Paternalistic leadership in four East Asian societies: generalizability and cultural differences of the triad model. Journal of CrossCultural Psychology, 45, 82-90.

de Mooij, M., \& Hofstede, G. (2002). Convergence and divergence in consumer behavior: implications for international retailing. Journal of Retailing, 78, 61-69.

Dellaert, B. G. C., \& Stremersch, S. (2005). Marketing mass-customized products: striking a balance between utility and complexity. Journal of Marketing Research, 42, 219-227.

Frank, B., Abulaiti, G., \& Enkawa, T. (2012). What characterizes Chinese consumer behavior? A crossindustry analysis of the Chinese diaspora in Japan. Marketing Letters, 23, 683-700.

Frank, B., Abulaiti, G., Torrico, B., \& Enkawa, T. (2013). How do Asia's two most important consumer markets differ? Japanese-Chinese differences in customer satisfaction and its formation. Journal of Business Research, 66, 2397-2405.

Greenleaf, E. A., \& Lehmann, D. R. (1995). Reasons for substantial delay in consumer decision making. Journal of Consumer Research, 22, 186-199.

Henrich, J., Heine, S. J., \& Norenzayan, A. (2010). The weirdest people in the world? Behavioral and Brain Sciences, 33, 61-83. 
Hildebrand, C., Häubl, G., Herrmann, A., \& Landwehr, J. R. (2013). When social media can be bad for you: community feedback stifles consumer creativity and reduces satisfaction with self-designed products. Information Systems Research, 24, 14-29.

Hildebrand, C., Häubl, G., \& Herrmann, A. (2014). Product customization via starting solutions. Journal of Marketing Research, 51, 707-725.

Hofmann, S. G., Asnaani, A., \& Hinton, D. E. (2010). Cultural aspects in social anxiety and social anxiety disorder. Depression and Anxiety, 27, 1117-1127.

Hofstede, G. (2001). Culture's consequences: comparing values, behaviors, institutions, and organizations across nations (2nd ed.). Thousand Oaks: Sage.

Hofstede, G. H., Hofstede, G. J., \& Minkov, M. (2010). Cultures and organizations: software of the mindintercultural cooperation and its importance for survival. New York: McGraw-Hill.

Hsu, F. L. (1971). Psychosocial homeostasis and jen: conceptual tools for advancing psychological anthropology. American Anthropologist, 73, 23-44.

Lehman, D. R., Chiu, C., \& Schaller, M. (2004). Psychology and culture. Annual Review of Psychology, 55, 689-714.

Lynn, M., \& Gelb, B. D. (1996). Identifying innovative national markets for technical consumer goods. International Marketing Review, 13, 43-57.

Markus, H. R., \& Kitayama, S. (1991). Culture and the self: implications for cognition, emotion, and motivation. Psychological Review, 98, 224-253.

Nisbett, R. E. (2003). The geography of thought. New York: Free Press.

Oyserman, D., Coon, H. M., \& Kemmelmeier, M. (2002). Rethinking individualism and collectivism: evaluation of theoretical assumptions and meta-analyses. Psychological Bulletin, 128, 3-72.

Preacher, K. J., \& Hayes, A. F. (2008). Asymptotic and resampling strategies for assessing and comparing indirect effects in multiple mediator models. Behavior Research Methods, 40, 879-891.

Quintal, V., Lee, J., \& Soutar, G. (2010). Tourists' information search: the differential impact of risk and uncertainty avoidance. International Journal of Tourism Research, 12, 321-333.

Schmitt, B. (2013). The changing face of the Asian consumer: insights and strategies for Asian markets. Singapore: McGraw-Hill.

Simonson, I. (2005). Determinants of customers' responses to customized offers: conceptual framework and research propositions. Journal of Marketing, 69, 32-45.

Singh, N., Zhao, H., \& Hu, X. (2005). Analyzing the cultural content of web sites: a cross-national comparison of China, India, Japan, and US. International Marketing Review, 22, 129-146.

Sismeiro, C., \& Bucklin, R. E. (2004). Modeling purchase behavior at an e-commerce web site: a taskcompletion approach. Journal of Marketing Research, 41, 306-323.

Steenkamp, J.-B. E. M., Hofstede, F. T., \& Wedel, M. (1999). A cross-national investigation into the individual and national cultural antecedents of consumer innovativeness. Journal of Marketing, 63, 55-69.

Valenzuela, A., Dhar, R., \& Zettelmeyer, F. (2009). Contingent response to self-customization procedures: implications for decision satisfaction and choice. Journal of Marketing Research, 46, 754-763.

van Everdingen, Y., \& Waarts, E. (2003). The effect of national culture on the adoption of innovations. Marketing Letters, 14, 217-232.

Yaveroglu, I. S., \& Donthu, N. (2002). Cultural influences on the diffusion of new products. Journal of International Consumer Marketing, 14, 49-63.

Yeniyurt, S., \& Townsend, J. (2003). Does culture explain acceptance of new products in a country? An empirical investigation. International Marketing Review, 20, 377-396. 